\title{
On Definition of Quantum Tomography via the Sobolev Embedding Theorem
}

\author{
Grigori Amosov*1 and Yakov Korennoy ${ }^{\dagger 2}$ \\ ${ }^{1}$ Steklov Mathematical Institute of Russian Academy of \\ Sciences, 8 Gubkina St., Moscow, 119991 Russia \\ ${ }^{2}$ Lebedev Physical Institute of Russian Academy of Sciences, \\ Leninskii prospect 53, Moscow 119991, Russia
}

August 20, 2019

\begin{abstract}
We obtain sufficient conditions on kernels of quantum states under which Wigner functions, optical quantum tomograms and linking their formulas are correctly defined. Our approach is based upon the Sobolev embedding theorem. The transition probability formula and the fractional Fourier transform are discussed in this framework.
\end{abstract}

2010 AMS Mathematical Subject Classification: 81P16, 46E35

Keywords: quantum tomography, Wigner function, optical tomogram, Sobolev embedding theorem, partial Fourier transform, Radon transform

\section{Introduction}

In [1] it was shown that the states of $n$-dimensional quantum systems can be completely described by the real and positive probability distribution functions of physical observable $\overline{\hat{x}}(\bar{\alpha})=\overline{\hat{q} \cos \alpha}+\overline{\hat{p} \sin \alpha}$, where $\overline{\hat{q} \cos \alpha}$ and $\overline{\hat{p} \sin \alpha}$ are $n$-dimensional vectors with the components $\hat{q}_{j} \cos \alpha_{j}$ and $\hat{q}_{j} \sin \alpha_{j}$. Here $\overline{\hat{q}}$ and $\overline{\hat{p}}$ are position and momentum operators, $\bar{\alpha}$ are angular parameters.

*gramos@mi-ras.ru

†abc772211@mail.ru 
Such a representation of quantum states is known as tomographic. In a variety of subsequent articles the properties of tomographic representation were investigated in detail (see, e.g. Review [2]).

The ordinary definition of optical quantum tomogram reads [1]

$$
\omega_{\hat{\rho}}(\bar{x}, \bar{\alpha})=\operatorname{Tr}\{\hat{\rho} \delta(\bar{x} \hat{I}-\overline{\hat{q} \cos \alpha}-\overline{\hat{p} \sin \alpha})\},
$$

where $\hat{\rho}$ is a density operator of the state.

Let us make sure that a trace in the righthand side of (11) is correctly defined for all $\hat{\rho}>0$ with $\operatorname{Tr}\{\hat{\rho}\}=1$ and the kernels $\rho\left(q, q^{\prime}\right) \in L_{2}\left(\mathbb{R}^{2 n}\right)$. Following to [3] let us define the characteristic function of a state $\hat{\rho}$ by the formula

$$
f_{\hat{\rho}}(\bar{x}, \bar{y})=\operatorname{Tr}\left\{\hat{\rho} e^{i(\bar{x} \cdot \overline{\hat{q}}+\bar{y} \cdot \overline{\hat{p}})}\right\} .
$$

It is known [3] that $f_{\hat{\rho}} \in L_{2}\left(\mathbb{R}^{2 n}\right) \cap C\left(\mathbb{R}^{2 n}\right)$. Hence we can define a function

$$
F_{\hat{\rho}}(\bar{t}, \bar{\alpha})=f_{\hat{\rho}}(\overline{t \cos \alpha}, \overline{t \sin \alpha}) .
$$

It follows from the inclusion $f_{\hat{\rho}} \in L_{2}\left(\mathbb{R}^{2 n}\right)$ that functions

$$
f_{\bar{s}, \bar{\alpha}}(\bar{t})=f_{\hat{\rho}}(\bar{s}+\overline{t \cos \alpha}, \bar{s}+\overline{t \sin \alpha}) \in L_{2}\left(\mathbb{R}^{n}\right)
$$

for almost all $\bar{s} \in \mathbb{R}^{n}$. Hence $F_{\hat{\rho}}(\cdot, \bar{\alpha}) \in L_{2}\left(\mathbb{R}^{n}\right)$ due to $f_{\hat{\rho}}$ is continuous. Formula (1) means that $\omega_{\hat{\rho}}(\cdot, \bar{\alpha})$ is the Fourier transform of $F_{\hat{\rho}}(\cdot, \bar{\alpha})$ and we see that it is correct as the Fourier transform of $L_{2}$-function.

It should be noted that given a characteristic function $f_{\hat{\rho}}$ it is possible to regenerate $\hat{\rho}$ in weak sense. Then, for a kernel of $\hat{\rho}$ in the coordinate representation we get [3]

$$
\rho\left(\bar{q}, \bar{q}^{\prime}\right)=\frac{1}{(2 \pi)^{n}} \int_{\mathbb{R}^{n}} e^{-\frac{i}{2}\left(\bar{q}+\bar{q}^{\prime}\right) \bar{y}} f_{\hat{\rho}}\left(\bar{q}-\bar{q}^{\prime}, \bar{y}\right) d \bar{y} .
$$

Thus, (3) is the partial Fourier transform of $f_{\rho}$. Hence we can find kernels $\rho_{\bar{\alpha}}(\cdot, \cdot) \in L^{2}\left(\mathbb{R}^{2 n}\right)$ of the operator $\hat{\rho}$ in integral representations associated with all the observables $\overline{\hat{x}}(\bar{\alpha})$. It results in a tomogram can be correctly defined by the formula

$$
\omega_{\hat{\rho}}(\bar{x}, \bar{\alpha})=\rho_{\bar{\alpha}}(\bar{x}, \bar{x})
$$

for any state $\hat{\rho}$, and $\omega_{\hat{\rho}}(\cdot, \bar{\alpha}) \in L_{1}\left(\mathbb{R}^{n}\right)$. Thus, formulae (1) and (4) give the same result for all states $\hat{\rho}$. Note that to take a trace in (4) we take into account that $\hat{\rho}>0$. For an arbitrary $\hat{\rho}$ of the trace class (4) is not valid. In our work, we will waive the requirement of positivity for $\hat{\rho}$. 
The situation becomes more complex for the Wigner function

$$
\mathcal{W}_{\hat{\rho}}(\bar{q}, \bar{p})=\frac{1}{(2 \pi)^{n}} \int_{\mathbb{R}^{2 n}} e^{i \bar{q} \cdot \bar{x}+i \bar{p} \cdot \bar{y}} f_{\hat{\rho}}(\bar{x}, \bar{y}) d^{n} \bar{x} d^{n} \bar{y} .
$$

The claim $f_{\hat{\rho}}(\cdot, \cdot) \in L_{2}\left(\mathbb{R}^{2 n}\right)$ results in $\mathcal{W}_{\hat{\rho}}(\cdot, \cdot) \in L_{2}\left(\mathbb{R}^{2 n}\right)$ but taking the Fourier transform we lose smoothness. Thus, the restriction of $\mathcal{W}_{\hat{\rho}}$ to a fixed hyperplane doesn't exist in general. This prevents the use of the Radon transform for $\mathcal{W}_{\hat{\rho}}$. To avoid these difficulties, articles concerning quantum tomography often deal only with a variety of bound states of quantum systems. In the case, wave functions as well as density matrixes of such states belong to the Schwartz spaces, i.e. $\psi(\cdot) \in \mathcal{S}\left(\mathbb{R}^{n}\right)$ and $\rho(\cdot, \cdot) \in \mathcal{S}\left(\mathbb{R}^{2 n}\right)$.

Up to the present time the question is opened about what class of functions do we need for the integral formulae connecting quantum tomograms and Wigner functions to be correct. In the present paper we will partially fill this gap using the famous Sobolev Embedding Theorem.

\section{Preliminaries}

Following [4] denote $\mathcal{S}\left(\mathbb{R}^{n}\right)$ the Schwartz space consisting of infinitely differentiable and fast descending at infinity functions $\psi(\bar{x})$ of $n$ variables $\bar{x}=$ $\left(x_{1}, \ldots, x_{n}\right)$. All functions $\psi \in \mathcal{S}\left(\mathbb{R}^{n}\right)$ are known to be summable with respect to any choice of variables $x_{j_{1}}, \ldots, x_{j_{k}}$. Moreover,

$$
\int_{\mathbb{R}^{k}} \psi(\bar{x}) d x_{j_{1}} \ldots d x_{j_{k}} \in \mathcal{S}\left(\mathbb{R}^{n-k}\right) .
$$

The Fourier transform is correctly defined for $\psi \in \mathcal{S}\left(\mathbb{R}^{n}\right)$ by the formula

$$
\mathcal{F}[\psi](\bar{x})=\frac{1}{(2 \pi)^{n}} \int_{\mathbb{R}^{n}} e^{-i \bar{x} \cdot \bar{y}} \psi(\bar{y}) d^{n} \bar{y} .
$$

It is known that $\mathcal{F}\left[\mathcal{S}\left(\mathbb{R}^{n}\right)\right]=\mathcal{S}\left(\mathbb{R}^{n}\right)$. Due to the Plancherel equality

$$
(2 \pi)^{n} \int_{\mathbb{R}^{n}}|\mathcal{F}[\psi](\bar{x})|^{2} d^{n} \bar{x}=\int_{\mathbb{R}^{n}}|\psi(\bar{y})|^{2} d^{n} \bar{y}
$$

the Fourier transform (6) can be extended to $L_{2}\left(\mathbb{R}^{n}\right)$. Fix the set of indices $J=\left\{j_{1}, \ldots, j_{k}\right\}, 0<k<n$, then also the partial Fourier transform $\mathcal{F}_{J}$ can be correctly defined on $\mathcal{S}\left(\mathbb{R}^{n}\right)$ as follows

$$
\mathcal{F}_{J}[\psi](\bar{x})=\left.\frac{1}{(2 \pi)^{k}} \int_{\mathbb{R}^{k}} e^{-i \sum_{j \in J} x_{j} y_{j}} \psi(\bar{y}) \prod_{j \in J} d y_{j}\right|_{\left\{y_{l}=x_{l}, l \in\{1, \ldots, n\} \backslash J\right\}} .
$$


Analogously to $\mathcal{F}$ taking into account the Plancherel equality we get that (77) can be extended to the space $L_{2}\left(\mathbb{R}^{k}\right)$.

Given $\nu>0$ denote $W_{2}^{\nu}\left(\mathbb{R}^{n}\right)$ the Sobolev space consisting of functions $\psi \in L_{2}\left(\mathbb{R}^{n}\right)$ such that the function $\tilde{\psi}(\bar{x})=|\bar{x}|^{\nu} \mathcal{F}[\psi](\bar{x})$ is square summable. If $\nu=k$ is an integer number, $W_{2}^{k}\left(\mathbb{R}^{n}\right)$ consists of functions possessing $k$ weak derivatives from $L_{2}\left(\mathbb{R}^{n}\right)$ in any variables. Let us consider the spaces

$$
\begin{gathered}
\mathcal{V}\left(\mathbb{R}^{2 n}\right)=W_{2}^{n+1}\left(\mathbb{R}^{2 n}\right) \cap \mathcal{F}\left[W_{2}^{n+1}\left(\mathbb{R}^{2 n}\right)\right], \\
\mathbb{V}\left(\mathbb{R}^{n}\right)=W_{2}^{n+1}\left(\mathbb{R}^{n}\right) \cap \mathcal{F}\left[W_{2}^{n+1}\left(\mathbb{R}^{n}\right)\right] .
\end{gathered}
$$

Lemma 1. The spaces $\mathcal{V}\left(\mathbb{R}^{2 n}\right)$ and $\mathbb{V}\left(\mathbb{R}^{n}\right)$ are invariant with respect to the Fourier transform and the partial Fourier transform as well.

Proof. The operator of multiplication by the variable $x_{j}$ is mapped to the differentiation $-i \frac{\partial}{\partial x_{j}}$ and vice versa. The result follows.

Given a function $f \in L_{2}\left(\mathbb{R}^{n}\right)$ one can try to define a function $F \in$ $L^{2}\left(\mathbb{R}^{m}\right), m<n$, which is a restriction of $F=\left.f\right|_{S}$ to some hyperplane $S$ with codimension $n-m$. Such the restriction $F$ known as a trace of $f$ is not defined in general. Our consideration is based upon the following famous statement [5, 6].

The Sobolev Embedding Theorem. Suppose that $f \in W_{2}^{\nu}\left(\mathbb{R}^{N}\right)$, then

$$
\text { (i) } f \in C^{\nu-\left[\frac{N}{2}\right]-1}\left(\mathbb{R}^{N}\right)
$$

([5]). Moreover, the trace $F=\left.f\right|_{S}$ to any hyperplane $S$ of codimension $N-m$ exists and

$$
\text { (ii) } \quad F \in W_{2}^{\nu-\frac{N-m}{2}}\left(\mathbb{R}^{m}\right)
$$

(Theorem 2 in [6]).

The lemma below shows why the space $\mathcal{V}\left(\mathbb{R}^{n}\right)$ can be useful in integral transformations.

Lemma 2. Given a function $f\left(x_{1}, \ldots x_{n}, y_{1}, \ldots y_{2}\right)$ belonging to $\mathcal{V}\left(\mathbb{R}^{2 n}\right)$ and a vector $\bar{\alpha} \in[0,2 \pi]^{n}$ the trace

$$
F_{\bar{\alpha}}\left(t_{1}, \ldots, t_{n}\right)=f\left(t_{1} \cos \alpha_{1}, \ldots, t_{n} \cos \alpha_{n}, t_{1} \sin \alpha_{1}, \ldots, t_{n} \sin \alpha_{n}\right)
$$

of $f$ to the hyperplane $x_{j} \sin \alpha_{j}-y_{j} \cos \alpha_{j}=0,1 \leq j \leq n$, is correctly defined and $F_{\bar{\alpha}} \in C\left(\mathbb{R}^{n}\right) \cap L_{1}\left(\mathbb{R}^{n}\right)$. 
Proof. Substituting $N=2 n, \nu=n+1$ and $m=n$ to Sobolev Embedding Theorem we obtain that $F_{\bar{\alpha}}$ is correctly defined and the inclusion $F_{\bar{\alpha}} \in W_{2}^{\frac{n}{2}+1}\left(\mathbb{R}^{n}\right)$ holds due to (ii). Then, (i) results in $F_{\bar{\alpha}} \in C\left(\mathbb{R}^{n}\right)$. Since $f \in \mathcal{V}\left(\mathbb{R}^{2 n}\right)$ we get $\mathcal{F}[f] \in W_{2}^{n+1}\left(\mathbb{R}^{2 n}\right)$. It gives rise $|\bar{x}|^{n+1} f(\bar{x})$ lies in $L_{2}\left(\mathbb{R}^{2 n}\right)$. Hence, $|\bar{t}|^{n+1} F_{\bar{\alpha}}(\bar{t})$ belongs to $L_{2}\left(\mathbb{R}^{n}\right)$. Applying the Schwartz inequality

$$
\begin{aligned}
& \left|\int_{\mathbb{R}^{n}} F_{\bar{\alpha}}\left(t_{1}, \ldots, t_{n}\right) d t_{1} \ldots d t_{n}\right|^{2} \\
& \quad \leq\left(\int_{\mathbb{R}^{n}} \prod_{j=1}^{n} \frac{1}{t_{j}^{2}+1} d t_{j}\right)\left(\int_{\mathbb{R}^{n}}\left|F_{\bar{\alpha}}\left(t_{1}, \ldots, t_{n}\right)\right|^{2} \prod_{j=1}^{n}\left(t_{j}^{2}+1\right) d t_{j}\right)
\end{aligned}
$$

we obtain $F_{\bar{\alpha}} \in L_{1}\left(\mathbb{R}^{n}\right)$.

Take a function $\rho(\cdot, \cdot) \in L_{2}\left(\mathbb{R}^{2 n}\right)$ and consider the integral operator $\hat{\rho}$ defined by the formula

$$
\hat{\rho}[\psi](\bar{x})=\int_{\mathbb{R}^{n}} \rho(\bar{x}, \bar{y}) \psi(\bar{y}) d^{n} \bar{y}, \psi \in L^{2}\left(\mathbb{R}^{n}\right) .
$$

If $\hat{\rho}$ belongs to the convex set $\mathfrak{S}$ consisting of hermitian and positive unit trace operators in $L_{2}\left(\mathbb{R}^{n}\right)$, it is called a quantum state. The important subclass of $\mathfrak{S}$ is pure quantum states $\hat{\rho}$ with the kernels $\rho(\bar{x}, \bar{y})=\xi(\bar{x}) \xi^{*}(\bar{y}), \xi \in$ $L_{2}\left(\mathbb{R}^{n}\right),\|\xi\|=1$. The characteristic function $f_{\hat{\rho}} \equiv f_{\rho}$ of $\hat{\rho} \in \mathfrak{S}$ is correctly defined by the formula (2). For a pure quantum state $\hat{\rho}=|\xi\rangle\langle\xi|$ we obtain

$$
f_{\xi}(\bar{x}, \bar{y})=\left\langle\xi\left|e^{i \bar{x} \cdot \overline{\hat{q}}+i \bar{y} \cdot \overline{\hat{p}}}\right| \xi\right\rangle .
$$

Using the Baker formula $e^{i x \hat{q}_{j}+i y \hat{p}_{j}}=e^{i x \hat{q}_{j}} e^{i y \hat{p}_{j}} e^{\frac{i x y}{2}}$ we can rewrite (2) as follows

$$
f_{\rho}(\bar{x}, \bar{y})=\int_{\mathbb{R}^{n}} e^{i \bar{x} \cdot \bar{t}} \rho\left(\bar{t}+\frac{\bar{y}}{2}, \bar{t}-\frac{\bar{y}}{2}\right) d^{n} \bar{t} .
$$

It follows from (11) that the map $\hat{\rho} \rightarrow f_{\rho}$ can be extended to the HilbertSchmidt operators $\hat{\rho}$ having the kernels $\rho(\cdot, \cdot) \in L_{2}\left(\mathbb{R}^{2 n}\right)$. In the case, $f_{\rho}(\cdot, \cdot) \in L_{2}\left(\mathbb{R}^{2 n}\right)[3]$.

Lemma 3. Formula (11) defines a linear map on the space $\mathcal{V}\left(\mathbb{R}^{2 n}\right)$. The invers transformation is given by the formula

$$
\rho\left(\bar{q}, \bar{q}^{\prime}\right)=\left.\frac{1}{(2 \pi)^{n}} \int_{\mathbb{R}^{n}} e^{-i \bar{x} \cdot \bar{t}} f_{\rho}(\bar{x}, \bar{y}) d^{n} \bar{x}\right|_{\bar{t}=\left(\bar{q}+\bar{q}^{\prime}\right) / 2, \bar{y}=\bar{q}-\bar{q}^{\prime}} .
$$


Proof. The nonsingular change of variables $\bar{q}=\bar{t}+\bar{y} / 2, \overline{q^{\prime}}=\bar{t}-\bar{y} / 2$ in the function $\rho\left(\bar{q}, \overline{q^{\prime}}\right)$ as well as the Fourier transform with respect to one of coordinates map $\mathcal{V}\left(\mathbb{R}^{2 n}\right)$ to itself. Applying the inverse Fourier transform to $f_{\hat{\rho}}(\bar{x}, \bar{y})$ with respect to $\bar{x}$ we obtain (12).

\section{Characteristic function, Wigner function and optical quantum tomogram}

Consider an integral operator $\hat{\rho}$ with the kernel $\rho(\cdot, \cdot) \in \mathcal{V}\left(\mathbb{R}^{2 n}\right)$. Then, Lemmas 1 and 3 result in the Wigner function (5) $\mathcal{W}_{\hat{\rho}} \equiv \mathcal{W}_{\rho}(\cdot, \cdot) \in \mathcal{V}\left(\mathbb{R}^{2 n}\right)$.

Obviously [1] the optical quantum tomogram is defined via the restriction of the partial Fourier transform of the characteristic function or the Radon transform [7] of the Wigner function. Nevertheless it can not be correctly done for an arbitrary $\rho(\cdot, \cdot) \in L_{2}\left(\mathbb{R}^{2 n}\right)$. Nevertheless, the application of Lemmas 2 and 3 shows that it is correct for kernels $\rho(\cdot, \cdot) \in \mathcal{V}\left(\mathbb{R}^{2 n}\right)$.

Theorem 1. If a kernel $\rho(\cdot, \cdot) \in \mathcal{V}\left(\mathbb{R}^{2 n}\right)$, then $f_{\rho}(\cdot) \in \mathcal{V}\left(\mathbb{R}^{2 n}\right)$ and $\mathcal{W}_{\rho}(\cdot, \cdot) \in$ $\mathcal{V}\left(\mathbb{R}^{2 n}\right)$ such that the following formulas are correct and define the same object called the optical quantum tomogram

$$
\begin{gathered}
\omega_{\rho}(\bar{x}, \bar{\alpha})=\frac{1}{(2 \pi)^{n}} \int_{\mathbb{R}^{n}} e^{-i \bar{x} \cdot \bar{t}} f_{\rho}(\overline{t \cos \alpha}, \overline{t \sin \alpha}) d^{n} \bar{t} \\
\omega_{\rho}(\bar{x}, \bar{\alpha})=\frac{1}{(2 \pi)^{n}} \int_{\mathbb{R}^{2 n}} \mathcal{W}_{\rho}(\bar{q}, \bar{p}) \delta(\bar{x}-\overline{q \cos \alpha}-\overline{p \sin \alpha}) d^{n} \bar{q} d^{n} \bar{p}
\end{gathered}
$$

where $\alpha_{j} \in[0,2 \pi], j \in\{1, \ldots, n\}$. Moreover, $\omega_{\rho}(\cdot, \bar{\alpha}) \in C\left(\mathbb{R}^{n}\right) \cap L_{1}\left(\mathbb{R}^{n}\right)$.

Proof. It follows from Lemma 1 that the characteristic function $f_{\rho} \in \mathcal{V}\left(\mathbb{R}^{2 n}\right)$. Let us make a change of variables

$$
\tilde{x}_{j}=x_{j} \cos \alpha_{j}+y_{j} \sin \alpha_{j}, \tilde{y}_{j}=x_{j} \sin \alpha_{j}-y_{j} \cos \alpha_{j}, 1 \leq j \leq n .
$$

Then, take the partial Fourier transform $\mathcal{F}_{\overline{\tilde{x}}}$ of $f_{\rho}(\overline{\tilde{x}}, \overline{\tilde{y}})$ with respect to variables $\overline{\tilde{x}}$. Due to Lemma $1 \mathcal{F}_{\tilde{x}}(f) \in \mathcal{V}\left(\mathbb{R}^{2 n}\right)$. Applying Lemma 2 we obtain that the trace $F_{\bar{\alpha}}$ of the function $\mathcal{F}_{\overline{\tilde{x}}}(f)$ with respect to the hyperplane $\tilde{y}_{j}=-x_{j} \sin \alpha_{j}+y_{j} \cos \alpha_{j}=0,1 \leq j \leq n$, is correctly defined and $F_{\bar{\alpha}}=$ $\omega_{\rho}(\cdot, \bar{\alpha}) \in C\left(\mathbb{R}^{n}\right) \cap L_{1}\left(\mathbb{R}^{n}\right)$. Since the Wigner function is the Fourier transform of the characteristic function we can conclude that $\mathcal{W}_{\rho}(\cdot, \cdot) \in \mathcal{V}\left(\mathbb{R}^{2 n}\right)$ due to Lemma 1. Taking into account Lemma 2 we obtain that the traces of $\mathcal{W}_{\rho}$ are determined for any hyperplane $-x_{j} \sin \alpha_{j}+y_{j} \cos \alpha_{j}=0,1 \leq j \leq n$. 
Applying the Radon transform to $\mathcal{W}_{\rho}(\bar{q}, \bar{p})$ we obtain (14). The coincidence of (13) and (14) follows from the Fourier slice theorem [8].

Remark. Transformation (13) is reversible with the inverse Fourier transform

$$
f_{\rho}(\overline{\lambda \cos \alpha}, \overline{\lambda \sin \alpha})=\int_{\mathbb{R}^{n}} e^{i \bar{x} \cdot \bar{\lambda}} \omega_{\rho}(\bar{x}, \bar{\alpha}) d^{n} \bar{x},
$$

and the change of variables from $\{(\bar{t}, \bar{\alpha})\}$ to the Cartesian coordinates

$$
\lambda_{j}=\operatorname{sgn}\left(y_{j}\right) \sqrt{x_{j}^{2}+y_{j}^{2}}, \quad \alpha_{j}=\cot ^{-1} \frac{x_{j}}{y_{j}}, \quad j \in\{1, \ldots, n\}
$$

gives us the characteristic function $f_{\rho}(\bar{x}, \bar{y})$, which can be transformed to the density matrix $\rho\left(\bar{q}, \bar{q}^{\prime}\right)$ with the help of formula (12).

\section{Transition probability between two states}

The transition probability $P_{12}$ between two states $\hat{\rho}_{1}$ and $\hat{\rho}_{2}$ of quantum system reads

$$
P_{12}=\int_{\mathbb{R}^{2 n}} \rho_{1}\left(\bar{q}, \overline{q^{\prime}}\right) \rho_{2}\left(\overline{q^{\prime}}, \bar{q}\right) d^{n} \bar{q} d^{n} \overline{q^{\prime}}
$$

The following theorem is valid.

Theorem 2. Given two optical tomograms $\omega_{\rho_{1}}(\bar{x}, \bar{\alpha})$ and $\omega_{\rho_{2}}(\bar{x}, \bar{\alpha})$ corresponding to two density matrixes $\rho_{1}\left(\bar{q}, \overline{q^{\prime}}\right) \in \mathcal{V}\left(\mathbb{R}^{2 n}\right)$ and $\rho_{2}\left(\bar{q}, \overline{q^{\prime}}\right) \in \mathcal{V}\left(\mathbb{R}^{2 n}\right)$ by means of formulas (11) and (13) we get

$$
P_{12}=\int_{[0 ; \pi]^{n} \times \mathbb{R}^{n}} d^{n} \bar{\alpha} d^{n} \bar{\lambda}\left(\prod_{j=1}^{n}\left|\lambda_{j}\right|\right) \int_{\mathbb{R}^{2 n}} d^{n} \bar{x} d^{n} \overline{x^{\prime}} e^{i \bar{\lambda} \cdot\left(\bar{x}-\overline{x^{\prime}}\right)} \omega_{\rho_{1}}(\bar{x}, \bar{\alpha}) \omega_{\rho_{2}}\left(\overline{x^{\prime}}, \bar{\alpha}\right) .
$$

Proof. Let us change the variables $\bar{q}=\bar{t}+\bar{y} / 2, \overline{q^{\prime}}=\bar{t}-\bar{y} / 2$ in the integral (16) and let us do the partial Fourier transforms of functions $\rho_{1}(\bar{t}+\bar{y} / 2, \bar{t}-\bar{y} / 2)$, $\rho_{2}(\bar{t}-\bar{y} / 2, \bar{t}+\bar{y} / 2)$ over the variables $\bar{t}$ taking into account Lemmas 1 and 3 . According to the Plancherel equality we get

$$
P_{12}=\int_{\mathbb{R}^{2 n}} f_{\rho_{1}}(\bar{x}, \bar{y}) f_{\rho_{2}}(-\bar{x},-\bar{y}) d^{n} \bar{x} d^{n} \bar{y}
$$


where $f_{\rho_{1}}(\bar{x}, \bar{y})$ and $f_{\rho_{2}}(-\bar{x},-\bar{y})$ are defined by (111).

The change of variables $x_{j}=\lambda_{j} \cos \alpha_{j}, y_{j}=\lambda_{j} \sin \alpha_{j}$ in the integral (18) gives rise to the following relation:

$$
P_{12}=\int_{[0 ; \pi]^{n} \times \mathbb{R}^{n}} d^{n} \bar{\alpha} d^{n} \bar{\lambda}\left(\prod_{j=1}^{n}\left|\lambda_{j}\right|\right) f_{\rho_{1}}(\overline{\lambda \cos \alpha}, \overline{\lambda \sin \alpha}) f_{\rho_{2}}(-\overline{\lambda \cos \alpha},-\overline{\lambda \sin \alpha}) .
$$

Due to Lemma 2 the functions $f_{\rho_{1}}(\overline{\lambda \cos \alpha}, \overline{\lambda \sin \alpha}), f_{\rho_{2}}(\overline{\lambda \cos \alpha}, \overline{\lambda \sin \alpha})$ belong to $L_{1}\left(\mathbb{R}^{n}\right)$ on $\bar{\lambda}$, and due to Theorem 1 the functions $\omega_{\rho_{1}}(\bar{x}, \bar{\alpha}), \omega_{\rho_{2}}(\bar{x}, \bar{\alpha})$ belong to $L_{1}\left(\mathbb{R}^{n}\right)$ on $\bar{x}$. Applying formula (15), which is the inverse Fourier transform of (13), we obtain the result (17).

\section{Fractional Fourier transforms of quantum states}

Using the Hamiltonians $\hat{H}_{j}=\frac{\hat{p}_{j}^{2}+\hat{q}_{j}^{2}}{2}$ let us define a unitary representation of the $n$th powers of the circle group $\mathbb{T}=[0,2 \pi]^{n}$ by the formula

$$
\mathbb{T} \ni \bar{\alpha} \rightarrow \hat{U}_{\bar{\alpha}}=\exp (-i \bar{\alpha} \cdot \overline{\hat{H}}) .
$$

It is straightforward to check that

$$
\hat{U}_{\bar{\alpha}}^{\dagger} \hat{q}_{j} \hat{U}_{\bar{\alpha}}=\hat{q}_{j} \cos \alpha_{j}+\hat{p}_{j} \sin \alpha_{j} .
$$

Given $\alpha \in(0 ; \pi)$ let us consider the unitary operator $\tilde{\mathcal{F}}_{\alpha}$ known as the fractional Fourier transform [9] and determined by the formula

$$
\tilde{\mathcal{F}}_{\alpha}^{q}[\varphi(q)](x)=\frac{\exp \left(\frac{i x^{2} \cos \alpha}{2 \sin \alpha}\right)}{\sqrt{2 \pi|\sin \alpha|}} \int_{\mathbb{R}} \exp \left(\frac{i q^{2} \cos \alpha}{2 \sin \alpha}-\frac{i x q}{\sin \alpha}\right) \varphi(q) d q .
$$

Using (20) we get 9

$$
\left|\hat{U}_{\bar{\alpha}}[\psi(\bar{q})](\bar{x})\right|^{2}=\left|\tilde{\mathcal{F}}_{\alpha_{1}}^{q_{1}} \circ \cdots \circ \tilde{\mathcal{F}}_{\alpha_{n}}^{q_{n}}[\psi(\bar{q})](\bar{x})\right|^{2},
$$

where $\tilde{\mathcal{F}}_{\alpha_{j}}^{q_{j}}$ acts on functions of the variable $q_{j}$.

Theorem 3. Suppose that $\rho(\bar{x}, \bar{y})=\psi(\bar{x}) \psi^{*}(\bar{y})$, where $\psi \in \mathbb{V}\left(\mathbb{R}^{n}\right)$. Then,

$$
\omega_{\rho}(\bar{x}, \bar{\alpha})=\left|\hat{U}_{\bar{\alpha}}[\psi](\bar{x})\right|^{2} .
$$


Proof. The space $\mathbb{V}\left(\mathbb{R}^{n}\right)$ can be alternatively described as $f \in \mathbb{V}\left(\mathbb{R}^{n}\right)$ iff

$$
\hat{q}_{1}^{s_{1}} q_{2}^{s_{2}} \cdots \hat{q}_{n}^{s_{n}} f, \hat{p}_{1}^{s_{1}} \hat{p}_{2}^{s_{2}} \cdots \hat{p}_{n}^{s_{n}} f \in L_{2}\left(\mathbb{R}^{n}\right)
$$

wherever integer powers $s_{j}, \sum_{j=1}^{n} s_{j} \leq n+1$. Hence, the property (19) results in $\hat{U}_{\bar{\alpha}} \mathbb{V}\left(\mathbb{R}^{n}\right)=\mathbb{V}\left(\mathbb{R}^{n}\right)$, and we obtain $\psi_{\bar{\alpha}} \equiv \hat{U}_{\bar{\alpha}} \psi \in \mathbb{V}\left(\mathbb{R}^{n}\right)$, where $\psi_{\bar{\alpha}}$ is a wave function of the state $\hat{\rho}$ in the representation of $\overline{\hat{x}}(\bar{\alpha})=\overline{\hat{q} \cos \alpha}+\overline{\hat{p} \sin \alpha}$. The condition $\psi \in \mathbb{V}\left(\mathbb{R}^{n}\right)$ is equivalent to $\rho \in \mathcal{V}\left(\mathbb{R}^{2 n}\right)$. Taking into account that $\omega_{\rho}(\bar{x}, \bar{\alpha})$ is a probability distribution corresponding to the observable $\overline{\hat{x}}(\bar{\alpha})$ in the state $\hat{\rho}$, we get the result. Note that $\omega_{\rho}(\cdot, \bar{\alpha}) \in L_{1}\left(\mathbb{R}^{n}\right) \cap C\left(\mathbb{R}^{n}\right)$ according to Theorem 1.

Remark. Analogously, given a density matrix of mixed state in the position representation $\rho\left(\bar{q}, \bar{q}^{\prime}\right) \in \mathcal{V}\left(\mathbb{R}^{2 n}\right)$ the corresponding density matrix $\rho_{\bar{\alpha}}\left(\bar{x}, \bar{x}^{\prime}\right)$ in the representation of $\overline{\hat{x}}(\bar{\alpha})$ equals

$$
\rho_{\bar{\alpha}}\left(\bar{x}, \bar{x}^{\prime}\right)=\left\langle\bar{x}\left|\hat{U}_{\bar{\alpha}} \hat{\rho} \hat{U}_{\bar{\alpha}}^{\dagger}\right| \bar{x}^{\prime}\right\rangle=\tilde{\mathcal{F}}_{\bar{\alpha}}^{\bar{q}}(\bar{x}) \circ \tilde{\mathcal{F}}_{-\bar{\alpha}}^{\bar{q}^{\prime}}\left(\bar{x}^{\prime}\right)\left[\rho\left(\bar{q}, \bar{q}^{\prime}\right)\right] .
$$

So, $\rho_{\bar{\alpha}}\left(\bar{x}, \bar{x}^{\prime}\right) \in \mathcal{V}\left(\mathbb{R}^{2 n}\right)$ according to Lemma 1 .

The tomogram $\omega_{\rho}(\bar{x}, \bar{\alpha})$ is a probability distribution of observable $\overline{\hat{x}}(\bar{\alpha})$ in the state $\hat{\rho}$, then $\omega_{\rho}(\bar{x}, \bar{\alpha})$ is the diagonal matrix element of $\rho_{\bar{\alpha}}\left(\bar{x}, \bar{x}^{\prime}\right)$, i.e. it is the restriction of $\rho_{\bar{\alpha}}\left(\bar{x}, \bar{x}^{\prime}\right)$ to the hyperplane $\bar{x}^{\prime}=\bar{x}$

$$
\omega_{\rho}(\bar{x}, \bar{\alpha})=\left.\rho_{\bar{\alpha}}\left(\bar{x}, \bar{x}^{\prime}\right)\right|_{\bar{x}^{\prime}=\bar{x}} .
$$

Moreover, $\omega_{\rho}(\cdot, \bar{\alpha}) \in C\left(\mathbb{R}^{n}\right) \cap L_{1}\left(\mathbb{R}^{n}\right)$, and formulas (21), (13), 14) define the same object called the optical quantum tomogram.

\section{Conclusion}

Our goal was to search for maximally common conditions on kernels of integral operators under which Wigner functions and optical quantum tomograms as well as linking their formulas are correctly defined. We have obtained sufficient conditions of this kind using the Sobolev Embedding theorem.

We defined the space $\mathcal{V}\left(\mathbb{R}^{2 n}\right)=W_{2}^{n+1}\left(\mathbb{R}^{2 n}\right) \cap \mathcal{F}\left[W_{2}^{n+1}\left(\mathbb{R}^{2 n}\right)\right]$ of kernels $\rho\left(\bar{q}, \bar{q}^{\prime}\right)$ for which the continuous and integrable tomograms $\omega_{\rho}(\bar{x}, \bar{\alpha})$ exist as well as the inverse transformations of these tomograms into original kernels. The space $\mathcal{V}\left(\mathbb{R}^{2 n}\right)$ is a subspace of the Sobolev space $W_{2}^{n+1}\left(\mathbb{R}^{2 n}\right)$ invariant with respect to to the Fourier transform, where $W_{2}^{n+1}\left(\mathbb{R}^{2 n}\right)$ consists 
of the functions belonging to the space $L_{2}\left(\mathbb{R}^{2 n}\right)$, having generalized derivatives of the order of $n+1$ such that $|\bar{x}|^{n+1} \mathcal{F}\left[\rho\left(\bar{q}, \bar{q}^{\prime}\right)\right](\bar{x}) \in L_{2}\left(\mathbb{R}^{2 n}\right)$. The space $\mathcal{V}\left(\mathbb{R}^{2 n}\right)$ is narrower than $L_{2}\left(\mathbb{R}^{2 n}\right)$ or $W_{2}^{n+1}\left(\mathbb{R}^{2 n}\right)$, but it is much wider than the Schwarz space $\mathcal{S}\left(\mathbb{R}^{2 n}\right)$. The main advantage of our approach is a justification of correctness for the integral formula linking optical quantum tomograms and Wigner functions by means of the Radon transform.

\section{References}

[1] S. Mancini, V. I. Manko, and P. Tombesi, "Symplectic tomography as classical approach to quantum systems", Phys. Lett. A, 2131 (1996).

[2] A. Ibort, V. I. Man'ko, G. Marmo, A. Simoni, and F. Ventriglia, "An introduction to the tomographic picture of quantum mechanics", Phys. Scr., 79, 065013 (2009).

[3] A. S. Holevo, Probabilistic and statistical aspects of quantum theory (Edizioni della Normale, 2011).

[4] M. Reed, B. Simon, Methods of Modern Mathematical Physics II. Fourier analysis, Self-Adjointness (Academic Press, 1975).

[5] S. Soboleff, "Sur un theoreme d'analyse fonctionnelle", Rec. Math. [Mat. Sbornik] N.S., 4(46):3 ( 471-497 (1938).

[6] S. M. Nikol'skii, "On imbedding, continuation and approximation theorems for differentiable functions of several variables", Russian Math. Surveys, 16:5, 55-104 (1961).

[7] J. Radon, "Uber die Bestimmung von Funktionen durch ihre Integralwerte langs gewisser Mannigfaltigkeiten", Ber. Verh. Sachs. Akad., 69, 262 (1917).

[8] S. Helgason, The Radon transform (Birkhauser, Boston, Basel, Stuttgart, 1980).

[9] V. Namias, "The fractional order Fourier transform and its application to quantum mechanics", J. Inst. Math. Appl., 25, 241-265 (1980). 